\title{
Criterion Stochastic Decision Making Method in the Tourism Industry
}

\author{
Vitaly Kostenko ${ }^{1, *}$, Volodymyr Kuznichenko² \& Volodymyr Lapshyn ${ }^{2}$ \\ ${ }^{1}$ Biochem Environmental Solutions Inc., 91 Milvan Drive, Toronto M9L 1Z7, Canada \\ ${ }^{2}$ Department of Mathematical Methods in Economics and Information Technologies, Kharkiv \\ Institute of Finances USUFIT, 5 Pletnivs'ky Lane, Kharkiv 61003, Ukraine \\ *Corresponding author: Biochem Environmental Solutions Inc., 91 Milvan Drive, Toronto \\ M9L 1Z7, Canada. Tel: 1-416-747-6600 ext.204 E-mail: vitaly_kostenko@yahoo.com
}

Received: March 3, 2018 Accepted: March 16, 2018 Published: March 25, 2018

doi: 10.5296/rae.v10i1.12761 URL: https://doi.org/10.5296/rae.v10i1.12761

\begin{abstract}
The development and application of effective and reliable decision making methods in the tourist business is an important problem. When choosing objects in the tourist business such as recreational territories, tourist routes and other attractions - the change in the number of objects, as well as in the number of choice criteria, is very important. Those who are tasked with making these sorts of decisions may find the method developed and proposed here - a criterion stochastic method - to be useful, on account of its universality and relative objectivity. When choosing an object (alternative) in a situation where their number - or the number of criteria - varies, the criterion stochastic method keeps the ratios of the initial global priorities of the alternatives (criteria) equal, as well as maintaining their comparison signs.
\end{abstract}

Keywords: tourism, decision making, criterion stochastic method 


\section{Introduction}

International tourism is an important part of the world market. Over the last several years, the tourism sector has experienced faster growth than all other sectors of the world economy. The tourism industry is a massive conglomeration of economic and social interests, and is an important element in most countries' economies. A healthy tourist industry relies on timely, motivated and well-planned decisions regarding its rules and development. The analysis of the various choices - and the use of dependable methods when making the decisions - is very important.

\section{Analysis of Recent Research and Publications}

The development of the tourism business, its problems and its prospects, as well as mathematical modeling of objects in the tourism industry, has been analysed in many publications (Reisinger Y., Viklyuk I., Melnichenko S., Sheenkova K.).

There are many decision making methods. The most commonly used method for choosing an optimal solution (alternative) based on chosen criteria in the absence of an objective measurement scale is the Analytic Hierarchy Process (AHP)(Saaty T.). It is still widely used in economics, including in the tourism sector.

Harichev V. estimated the efficiency factors of market management of a tourism enterprise when using the AHP. When applying the method of expert opinions, Maksymyuk N. determined the external and internal factors that have an effect on a tourism enterprise's efficiency. The procedure for choosing the optimal functional diagrams of zonal territories using the AHP was investigated by Kozar V., Kozar L. and Ilchenko T.

Moiseeva T. and Wissarionova L. a detailed hierarchical analysis of three ecological paths on the territory of the National Park"Paanayarvi" was carried out: the Mäntykoski and Kivakakkoski waterfall trails and the Kivakkatunturi mountain trail. The analysis showed that the path most interesting to tourists was the Mäntykoski waterfall trail. The most important criterion turned out to be aesthetic value. When choosing an object (alternative) in the tourism business, it's not uncommon to experience a change in their number, as well as a change in the number of criteria. In these cases, the use of the AHP can lead to changing initial global priorities of the alternatives (criteria). Furthermore, there will necessarily be a change in their ratios, which makes decision making more difficult.

The method of analytically structuring a series of alternatives and criteria (APM - analytical procedure method) and the criterion stochastic analytical decision making method (CSM criterion stochastic method) were developed by Kuznichenko V. and Lapshyn V. These methods preserve the initial distributions and ratios of the global priorities when the number of alternatives (criteria) changes. 


\section{The Purpose}

The purpose of this article is the application of the criterion stochastic method and the demonstration of its advantages during the decision making process in the tourism industry.

\section{Results}

Let's look at the decision making process when picking an object in the tourism industry (recreational territories, tourist routes, or other tourist attractions). Let's say that we are picking out of three objects (alternatives) $\left(A_{1}, A_{2}, A_{3}\right)$ in accordance with three selected criteria $\left(K_{1}, K_{2}, K_{3}\right)$.

When a quantitative comparison is impossible, the nine-point scale from Saaty $\mathrm{T}$. is used. This scale is written in the following way: equal importance $-1: 1$, small advantage $-3: 1$, moderate advantage $-5: 1$, significant advantage $-7: 1$, absolute advantage $-9: 1(2,4,6$ and 8 are intermediate advantage values). Let's construct the pair comparison matrices for the criteria $K_{1}, K_{2}, K_{3}$ and alternatives $A_{1}, A_{2}, A_{3}$.

Each line contains the expert pair comparison values (weights) of one structural unit relative to the others. This procedure is carried out for all of the lines. We note that the tables (for one expert) correspond to inverse symmetric matrices $\left(A=\left\{a_{I J}\right\}, i, j=\overline{1, n}\right)$, meaning that their elements satisfy the following condition:

$$
a_{i j}=\frac{1}{a_{j i}}
$$

where $a_{i j}$ is the matrix element ( $i$ is the line number and $j$ is the column number, both in the table and in the corresponding matrix). The eigenvectors $V\left(Z_{i}\right)(Z=A, K)$ of the pair comparison matrices (the final column of the table) are defined in the following way:

$$
V(Z)=\frac{\sum_{j=1}^{n} a_{i j}}{\sum_{i, j=1}^{n} a_{i j}}
$$

In order to determine the global priorities of the alternatives, it is sufficient to compare the alternatives to all of the criteria and to compare the criteria to one alternative (or vice versa). The other tables are reconstructed using the relative weight proportions.

We choose to compare $A_{1}, A_{2}, A_{3}$ to the criteria and $K_{1}, K_{2}, K_{3}$ to the alternative $A_{1}$. The results of these pair comparisons can be found in Tables 1-4. 
Table 1. Pair Comparison Matrix of Alternatives in Relation to the Criterion

\begin{tabular}{lllll}
\hline$K_{1}$ & $A_{1}$ & $A_{2}$ & $A_{3}$ & \multicolumn{1}{c}{$V\left(K_{1}\right)$} \\
\hline$A_{1}$ & 1 & $1 / 6$ & $1 / 8$ & $1 / 15$ \\
$A_{2}$ & 6 & 1 & $3 / 4$ & $2 / 5$ \\
$A_{3}$ & 8 & $4 / 3$ & 1 & $8 / 15$ \\
\hline
\end{tabular}

Table 2. Pair Comparison Matrix of Alternatives in Relation to the Criterion

\begin{tabular}{lllll}
\hline$K_{2}$ & $A_{1}$ & $A_{2}$ & $A_{3}$ & $V\left(K_{2}\right)$ \\
\hline$A_{1}$ & 1 & $3 / 2$ & 3 & $1 / 2$ \\
$A_{2}$ & $2 / 3$ & 1 & 2 & $1 / 3$ \\
$A_{3}$ & $1 / 3$ & $1 / 2$ & 1 & $1 / 6$ \\
\hline
\end{tabular}

Table 3. Pair Comparison Matrix of Alternatives in Relation to the Criterion

\begin{tabular}{ccccc}
\hline$K_{3}$ & $A_{1}$ & $A_{2}$ & $A_{3}$ & $V\left(K_{3}\right)$ \\
\hline$A_{1}$ & 1 & $7 / 8$ & $7 / 6$ & $1 / 3$ \\
$A_{2}$ & $8 / 7$ & 1 & $4 / 3$ & $8 / 21$ \\
$A_{3}$ & $6 / 7$ & $3 / 4$ & 1 & $2 / 7$ \\
\hline
\end{tabular}

Table 4. Pair Comparison Matrix of Criteria in Relation to the Alternative

\begin{tabular}{ccccc}
\hline$A_{1}$ & $K_{1}$ & $K_{2}$ & $K_{3}$ & $V\left(A_{1}\right)$ \\
\hline$K_{1}$ & 1 & $1 / 6$ & $1 / 7$ & $1 / 14$ \\
$K_{2}$ & 6 & 1 & $6 / 7$ & $3 / 7$ \\
$K_{3}$ & 7 & $7 / 6$ & 1 & $1 / 2$ \\
\hline
\end{tabular}

The numerical values in Tables 1-4 indicate the ratios of the weights $x_{i j}$ of the alternatives $\left(A_{i}\right)$ and the criteria $\left(K_{j}\right)$, which were determined in the pair comparison (weighing) process. For instance, in Table 4 , the first line (without $V\left(A_{1}\right)$ ) means $\left(x_{11} / x_{11}\right),\left(x_{11} / x_{12}\right)$, $\left(x_{11} / x_{13}\right)$.

Tables 5 and 6 are filled in using values from Tables 1-4. For instance, in Table 5, 
$a_{12}=\left(x_{21} / x_{22}\right)=\left(x_{12} / x_{22}\right) *\left(x_{11} / x_{12}\right) *\left(x_{21} / x_{11}\right)=3 / 2 \quad\left(\left(x_{12} / x_{22}\right)=3 / 2\right)$ is the element $a_{12}$ from Table $2 ;\left(x_{11} / x_{12}\right)=1 / 6$ is $a_{12}$ from Table $4 ;\left(x_{11} / x_{12}\right)=6$ is $a_{21}$ from Table 1).

Table 5. Pair Comparison Matrix of Criteria in Relation to the Alternative

\begin{tabular}{lllll}
\hline$A_{2}$ & $K_{1}$ & $K_{2}$ & $K_{3}$ & $V\left(A_{2}\right)$ \\
\hline$K_{1}$ & 1 & $3 / 2$ & $3 / 4$ & $1 / 3$ \\
$K_{2}$ & $2 / 3$ & 1 & $1 / 2$ & $2 / 9$ \\
$K_{3}$ & $4 / 3$ & 2 & 1 & $4 / 9$ \\
\hline
\end{tabular}

Table 6. Pair Comparison Matrix of Criteria in Relation to the Alternative

\begin{tabular}{lllll}
\hline$A_{3}$ & $K_{1}$ & $K_{2}$ & $K_{3}$ & $V\left(A_{3}\right)$ \\
\hline$K_{1}$ & 1 & 4 & $4 / 3$ & $1 / 2$ \\
$K_{2}$ & $1 / 4$ & 1 & $1 / 3$ & $1 / 8$ \\
$K_{3}$ & $3 / 4$ & 3 & 1 & $3 / 8$
\end{tabular}

In the CSM, the next step is finding the matrices $C(A)$ and $C(K)$ :

$$
C(A)=V^{T}(A) * V^{T}(K), C(K)=V^{T}(K) * V^{T}(A)
$$

Here, $V^{T}(A)$ and $V^{T}(K)$ are matrices which are built line-by-line using the eigenvectors of the matrices/tables 1-6.

$$
\begin{aligned}
& C(A)=\left(\begin{array}{ccc}
\frac{1}{14} & \frac{3}{7} & \frac{1}{2} \\
\frac{1}{3} & \frac{2}{9} & \frac{4}{9} \\
\frac{1}{2} & \frac{1}{8} & \frac{3}{8}
\end{array}\right) *\left(\begin{array}{ccc}
\frac{1}{15} & \frac{2}{5} & \frac{8}{15} \\
\frac{1}{2} & \frac{1}{3} & \frac{1}{6} \\
\frac{1}{3} & \frac{8}{21} & \frac{2}{7}
\end{array}\right)=\left(\begin{array}{ccc}
\frac{27}{70} & \frac{38}{105} & \frac{53}{210} \\
\frac{38}{135} & \frac{356}{945} & \frac{323}{945} \\
\frac{53}{240} & \frac{323}{840} & \frac{221}{560}
\end{array}\right) \\
& C(A)=\left(\begin{array}{ccc}
\frac{1}{15} & \frac{2}{5} & \frac{8}{15} \\
\frac{1}{2} & \frac{1}{3} & \frac{1}{6} \\
\frac{1}{3} & \frac{8}{21} & \frac{2}{7}
\end{array}\right) *\left(\begin{array}{ccc}
\frac{1}{14} & \frac{3}{7} & \frac{1}{2} \\
\frac{1}{3} & \frac{2}{9} & \frac{4}{9} \\
\frac{1}{2} & \frac{1}{8} & \frac{3}{8}
\end{array}\right)=\left(\begin{array}{ccc}
\frac{17}{42} & \frac{58}{315} & \frac{37}{90} \\
\frac{29}{126} & \frac{111}{359} & \frac{199}{432} \\
\frac{37}{126} & \frac{199}{756} & \frac{335}{756}
\end{array}\right)
\end{aligned}
$$


From the systems of equations $(m=n=3)$

$$
\begin{gathered}
\left\{\begin{array}{c}
\bar{W}(A)=\bar{W}(A) * C(A) \\
\sum_{i=1}^{m} W\left(a_{i}\right)=1
\end{array}\right. \\
\left\{\begin{array}{c}
\bar{W}(K)=\bar{W}(K) * C(K) \\
\sum_{j=1}^{n} W\left(k_{j}\right)=1
\end{array}\right.
\end{gathered}
$$

We obtain the global priorities of the alternatives $\bar{W}(A)$ and of the criteria $\bar{W}(K)$. The components of the global priorities are equal to

$$
\bar{W}(A)=\left(\frac{7}{24}, \frac{3}{8}, \frac{1}{3}\right), \bar{W}(K)=\left(\frac{5}{16}, \frac{1}{4}, \frac{7}{16}\right)
$$

and

satisfy

the

following

inequalities:

$W\left(A_{2}\right)>W\left(A_{3}\right)>W\left(A_{1}\right) ; W\left(K_{3}\right)>W\left(K_{1}\right)>W\left(K_{2}\right)$.

Let's add another object to the analysis. Then, we add the alternative $A_{4}$ to Tables 1-3. Tables 4-6 remain unchanged. The results of the pair comparisons are presented in Tables 7-9.

Table 7. Pair Comparison Matrix of Alternatives in Relation to the Criterion

\begin{tabular}{ccccccc}
\hline$K_{1}$ & $A_{1}$ & $A_{2}$ & $A_{3}$ & $A_{4}$ & $V\left(K_{1}\right)$ \\
\hline$A_{1}$ & 1 & $1 / 6$ & $1 / 8$ & $1 / 5$ & $1 / 20$ \\
$A_{2}$ & 6 & 1 & $3 / 4$ & $6 / 5$ & $3 / 10$ \\
$A_{3}$ & 8 & $4 / 3$ & 1 & $8 / 5$ & $2 / 5$ \\
$A_{4}$ & 5 & $5 / 6$ & $5 / 8$ & 1 & $1 / 4$ \\
\hline
\end{tabular}

Table 8. Pair comparison Matrix of Alternatives in Relation to the Criterion

\begin{tabular}{cccccc}
\hline$K_{2}$ & $A_{1}$ & $A_{2}$ & $A_{3}$ & $A_{4}$ & $V\left(K_{2}\right)$ \\
\hline$A_{1}$ & 1 & $3 / 2$ & 3 & $1 / 2$ & $1 / 4$ \\
$A_{2}$ & $2 / 3$ & 1 & 2 & 1 & $1 / 6$ \\
$A_{3}$ & $1 / 3$ & $1 / 2$ & 1 & $1 / 2$ & $1 / 12$ \\
$A_{4}$ & $2 / 3$ & 1 & 2 & 1 & $1 / 2$ \\
\hline
\end{tabular}


Table 9. Pair Comparison Matrix of Alternatives in Relation to the Criterion

\begin{tabular}{cccccc}
\hline$K_{3}$ & $A_{1}$ & $A_{2}$ & $A_{3}$ & $A_{4}$ & $V\left(K_{3}\right)$ \\
\hline$A_{1}$ & 1 & $7 / 8$ & $7 / 6$ & $7 / 3$ & $7 / 24$ \\
$A_{2}$ & $8 / 7$ & 1 & $4 / 3$ & $8 / 3$ & $1 / 3$ \\
$A_{3}$ & $6 / 7$ & $3 / 4$ & 1 & 2 & $1 / 4$ \\
$A_{4}$ & $3 / 7$ & $3 / 8$ & $1 / 2$ & 1 & $1 / 8$ \\
\hline
\end{tabular}

Table 10 is formed using Tables 7-9 and 4.

Table 10. Pair Comparison Matrix of Criteria in Relation to the Alternative

\begin{tabular}{ccccc}
\hline$A_{4}$ & $K_{1}$ & $K_{2}$ & $K_{3}$ & $V\left(A_{4}\right)$ \\
\hline$K_{1}$ & 1 & $5 / 4$ & $5 / 3$ & $5 / 12$ \\
$K_{2}$ & $4 / 5$ & 1 & $4 / 3$ & $1 / 3$ \\
$K_{3}$ & $3 / 5$ & $3 / 4$ & 1 & $1 / 4$ \\
\hline
\end{tabular}

For the case under consideration, the matrices $C(A)$ and $C(K)$ are:

$$
\begin{gathered}
C(A)=\left(\begin{array}{lll}
\frac{1}{14} & \frac{3}{7} & \frac{1}{2} \\
\frac{1}{3} & \frac{2}{9} & \frac{4}{9} \\
\frac{1}{2} & \frac{1}{8} & \frac{3}{8} \\
\frac{1}{4} & \frac{3}{5} & \frac{3}{20}
\end{array}\right) *\left(\begin{array}{cccc}
\frac{1}{20} & \frac{3}{10} & \frac{2}{5} & \frac{1}{4} \\
\frac{1}{4} & \frac{1}{6} & \frac{1}{12} & \frac{1}{2} \\
\frac{7}{24} & \frac{1}{3} & \frac{1}{4} & \frac{1}{8}
\end{array}\right)=\left(\begin{array}{cccc}
\frac{49}{191} & \frac{109}{420} & \frac{53}{280} & \frac{33}{112} \\
\frac{109}{540} & \frac{77}{270} & \frac{71}{270} & \frac{1}{4} \\
\frac{53}{320} & \frac{7}{240} & \frac{73}{240} & \frac{15}{64} \\
\frac{33}{160} & \frac{9}{40} & \frac{3}{16} & \frac{61}{160}
\end{array}\right), \\
C(K)=\left(\begin{array}{cccc}
\frac{1}{20} & \frac{3}{10} & \frac{2}{5} & \frac{1}{4} \\
\frac{1}{4} & \frac{1}{6} & \frac{1}{12} & \frac{1}{2} \\
\frac{7}{24} & \frac{1}{3} & \frac{1}{4} & \frac{1}{8}
\end{array}\right) *\left(\begin{array}{ccc}
\frac{1}{14} & \frac{3}{7} & \frac{1}{2} \\
\frac{1}{3} & \frac{2}{9} & \frac{4}{9} \\
\frac{1}{2} & \frac{1}{8} & \frac{3}{8} \\
\frac{1}{4} & \frac{3}{5} & \frac{3}{20}
\end{array}\right)=\left(\begin{array}{ccc}
\frac{41}{112} & \frac{121}{420} & \frac{83}{240} \\
\frac{112}{503} & \frac{5}{11} & \frac{109}{357} \\
\frac{83}{288} & \frac{109}{357} & \frac{163}{401}
\end{array}\right),
\end{gathered}
$$

The global priorities of the alternatives $\bar{W}(A)$ and of the criteria $\bar{W}(K)$ are obtained from the systems of equations (6), (7) $(m=4, n=3)$ :

$$
\bar{W}(A)=\left(\frac{7}{34}, \frac{9}{34}, \frac{4}{17}, \frac{5}{17}\right), \bar{W}(K)=\left(\frac{5}{17}, \frac{6}{17}, \frac{6}{17}\right) .
$$

The alternative $A_{4}$ occupied the fourth position (depending on the pair comparisons, it could 
have occupied any position), but the ratios between the first three remained constant: $W\left(A_{2}\right): W\left(A_{3}\right): W\left(A_{1}\right)=9: 8: 7$.

If the investor, when choosing among three objects, decides to add another criterion $K_{4}$, then the expert will use pair comparisons to create Tables 11 and 12.

Table 11. Pair Comparison Matrix of Alternatives in Relation to the Criterion

\begin{tabular}{ccccc}
\hline$K_{3}$ & $A_{1}$ & $A_{2}$ & $A_{3}$ & $V\left(K_{3}\right)$ \\
\hline$A_{1}$ & 1 & $1 / 7$ & $1 / 4$ & $1 / 12$ \\
$A_{2}$ & 7 & 1 & $7 / 4$ & $7 / 12$ \\
$A_{3}$ & 4 & $4 / 7$ & 1 & $4 / 12$ \\
\hline
\end{tabular}

Table 12. Pair Comparison Matrix of Criteria in Relation to the Alternative

\begin{tabular}{cccccc}
\hline$A_{1}$ & $K_{1}$ & $K_{2}$ & $K_{3}$ & $K_{4}$ & $V\left(A_{1}\right)$ \\
\hline$K_{1}$ & 1 & $1 / 6$ & $1 / 7$ & 1 & $1 / 15$ \\
$K_{2}$ & 6 & 1 & $6 / 7$ & 6 & $6 / 15$ \\
$K_{3}$ & 7 & $7 / 6$ & 1 & 7 & $7 / 15$ \\
$K_{4}$ & 1 & $1 / 6$ & $1 / 7$ & 1 & $1 / 15$ \\
\hline
\end{tabular}

The global priorities of the alternatives $\bar{W}(A)$ and of the criteria $\bar{W}(K)$ are obtained from formulas (3) and from the systems of equations (6), (7) $(m=3, n=4)$ :

$$
\bar{W}(A)=\left(\frac{1}{4}, \frac{5}{12}, \frac{1}{3}\right), \bar{W}(K)=\left(\frac{1}{4}, \frac{1}{5}, \frac{7}{20}, \frac{1}{5}\right)
$$

A comparison with the values from formula (11) shows that the fourth criterion has the same weight as the second and a smaller weight than the third and first: $W\left(K_{3}\right)>W\left(K_{1}\right)>W\left(K_{2}\right)=W\left(K_{4}\right)$. The ratios between the criteria remained constant: $W\left(K_{3}\right): W\left(K_{1}\right): W\left(K_{2}\right)=7: 5: 4$.

Let's compare the CSM and AHP in the context of the current case. In the AHP approach, one must construct a criterion pair comparison matrix with respect to the goal (the choice of the optimal object). Let's say that according to expert opinions, it has the form seen in Table 13: 


\section{Macrothink}

Table 13. Criterion Pair Comparison Matrix with Respect to the Goal

\begin{tabular}{ccccc}
\hline$G$ & $K_{1}$ & $K_{2}$ & $K_{3}$ & $V(G)$ \\
\hline$K_{1}$ & 1 & $1 / 3$ & $1 / 2$ & $1 / 6$ \\
$K_{2}$ & 3 & 1 & $3 / 2$ & $3 / 6$ \\
$K_{3}$ & 2 & $2 / 3$ & 1 & $2 / 6$ \\
\hline
\end{tabular}

The global priorities of the alternatives $A_{1}, A_{2}, A_{3}$ are determined by multiplying two matrices. The first is the matrix whose columns contain the eigenvectors $V\left(K_{i}\right),(i=\overline{1,3})$, while the second is simply the vector-column $\mathrm{V}(\mathrm{G})$ :

$$
W(A)=\left(\begin{array}{ccc}
\frac{1}{15} & \frac{1}{2} & \frac{1}{3} \\
\frac{2}{5} & \frac{1}{3} & \frac{8}{21} \\
\frac{8}{15} & \frac{1}{6} & \frac{2}{7}
\end{array}\right) *\left(\begin{array}{c}
\frac{1}{6} \\
\frac{3}{6} \\
\frac{2}{6}
\end{array}\right)=\left(\begin{array}{c}
\frac{469}{1260} \\
\frac{454}{1260} \\
\frac{337}{1260}
\end{array}\right)
$$

The global priorities of the alternatives obey the relation $W\left(K_{1}\right) / W\left(K_{2}\right)>W\left(K_{3}\right)$.

When adding yet another object to the analysis, the global priorities of the alternatives $A_{1}, A_{2}, A_{3}, A_{4}$, when using $V\left(K_{i}\right),(i=\overline{1,3})$ from Tables 7-9 and $V(G)$ from Table 13, are determined in a way that is analogous to formula (13):

$$
W(A)=\left(\begin{array}{ccc}
\frac{1}{20} & \frac{1}{4} & \frac{7}{24} \\
\frac{3}{10} & \frac{1}{6} & \frac{1}{3} \\
\frac{2}{5} & \frac{1}{12} & \frac{1}{4} \\
\frac{1}{4} & \frac{1}{2} & \frac{1}{8}
\end{array}\right) *\left(\begin{array}{l}
\frac{1}{6} \\
\frac{3}{6} \\
\frac{2}{6}
\end{array}\right)=\left(\begin{array}{c}
\frac{83}{360} \\
\frac{88}{360} \\
\frac{69}{360} \\
\frac{120}{360}
\end{array}\right)
$$

In this event, the relation $W\left(K_{4}\right)>W\left(K_{2}\right)>W\left(K_{1}\right)>W\left(K_{3}\right)$ is obeyed.

As we see, the addition of the alternative $\mathrm{A}_{4}$, in the AHP approach, has led to a change of the global priorities.

We would also like to note that the level of pair comparison of the criteria with respect to the goal can, as a rule, lead to an inconsistency between the criteria and alternatives. The results of the AHP (Formula 14) differ from the CSM results even without the consideration of additional objects. 


\section{Conclusions}

The proposed criterion stochastic method can be a universal and relatively objective decision making method in the tourism industry. For someone who is making decisions, the logical nature of the method is very important, and so the ratios and relative positions between the global priorities of the alternatives, which remain after they change in number during the decision making process, need to remain constant. When the number of alternatives (criteria) changes, the criterion stochastic method keeps the ratios of the initial global priorities of the alternatives (criteria) equal, as well as maintaining their comparison signs. The same cannot be said for the AHP - there, the ratios between the alternatives can change, which complicates decision-making. The CSM and APM, when constructing the pair comparison matrices, use similar and more objective methods that are rooted in the characteristics of the objects themselves, unlike the AHP, which uses the more subjective pair comparisons of the criteria with respect to a goal (this is absent in the CSM and APM). In the CSM, the global priorities of the alternatives (criteria) are obtained from the equilibrium state of a Markov chain system. In this event, a step-by-step correction procedure of the initial matrices $C(A)$ and $C(K)$ is carried out. Unlike the AHP, the method developed here defines the global priorities of the alternatives and criteria in a self-consistent manner.

Subsequent analyses may focus on expanding the applications of the proposed method to multi-level economic systems.

\section{References}

Harichev, V. (2009). The using of the method of analysis of hierarchies for the estimation of the factors which provide the effectiveness of marketing management of tourist enterprises. Management an economy: theory and practice: Collection of scientific papers. DIIENASU, 1, 224-234.

Kostenko, E., Kuznichenko, V., \& Lapshyn, V. (2014). Comparison of Decision-Making Methods. Research in Applied Economics, 6(3), 17-27. https://doi.org/10.5296/rae.v6i3.5704

Kozar, V., Kozar, L., \& Ilchenko, T. (2011). Using analysis method of hierarchies for decision of task of a plan organization of city territory. Transactions of Kremenchuk Mykhailo Ostrohradskyi National University, 4(69), 111-116.

Kuznichenko, V., \& Lapshyn, V. (2014). Methods of making decision on the basis of pair comparisons: Decision of tasks of theory of choice and making decision at many criteria on the basis of pair comparisons. Saarbrücken, Deutschland: Palmarium academic publishing. p. 63.

Maksymyuk, N. (2011). Factor impact assessment on efficiency of activity of tourism industry enterprises in the Crimea. Scientific Notes of Taurida National V.I. Vernadsky University: Economy and Management, 24(63), 123-131.

Melnichenko, S., \& Sheenkova, K. (2015). Management business processes in tourism. Kyiv, Ukraine: KNUTE. p. 264.

Moiseeva, T., \& Wissarionova, L. (2014). Application of hierarchy analysis approach to the 
characterization of eco-trails in the National Park "Paanayarvi". Principy ekologii, 3(1), 15-24. https://doi.org/10.15393/j1.art.2014.3261

Reisinger, Y. (2009). International Tourism Cultures and Behaviour. Amsterdam: Butterworth-Heinemann, Elsevier LTD. p. 217.

Reisinger, Y. (2013). Transformational Tourism: Tourist Perspectives. Kuwait: GUST. p. 137. https://dx.doi.org/10.1079/9781780642093.0000

Saaty, T. (1989). Making decision: the analytic hierarchy process. Moscow: Radio and communication. p. 316.

Saaty, T. (1990). How to make a decision: The analytic hierarchy process. European Journal of Operational Research, 48(1), 9-26. https://doi.org/10.1016/0377-2217(90)90057-I

Viklyuk, Ya. (2010). Mathematical design of objects of tourist industry. Chernivtsi, Ukraine: Books - XXI. p. 340.

\section{Copyright Disclaimer}

Copyright for this article is retained by the author(s), with first publication rights granted to the journal.

This is an open-access article distributed under the terms and conditions of the Creative Commons Attribution license (http://creativecommons.org/licenses/by/3.0/). 